\title{
Using Molinspiration as a didactic complement into teaching subjects of Medicinal Chemistry
}

\author{
Custódia Fonseca
}

Medicinal Chemistry has high rates of failure and abandonment at the beginning of the courses where this disciplines are taught, as occurs in pharmaceutical sciences.

In University of Algarve (UAlg), there has been an effort to avoid school failure in these topics by increasing student motivation, a very important issue for the students get success.

For this has been used in teaching Medicinal Chemistry, the web application called Molinspiration. This website/tool allows one to input a chemical structure and then after, view predicted properties of structure and its bioactivity against six different common drug targets. Using this application, students are transported to a virtual laboratory which making easier to understand and practice the basic scientific knowledge that has been previously explained in theoretical classes.

The virtual labs implemented follow the common steps described below:

1- The teacher explained how the application Molinspiration works

2- It were proposed to the students carry out a series of works related to the topics that had been explained in the teaching classes

3- The students formed groups for discussion the performed activities.

These experiences were highly appreciated by the students and increased their motivation to the subjects studied, and the results obtained were improved. 